\title{
Reversible posterior leukoencephalopathy syndrome secondary to systemic-onset juvenile idiopathic arthritis: A case report and review of the literature
}

\author{
PINGPING ZHANG ${ }^{1,2}$, XIAOFENG LI ${ }^{2}$, YATING LI ${ }^{2}$, JING WANG ${ }^{1}$, HUASONG ZENG $^{1}$ and XIAOFENG ZENG ${ }^{3}$ \\ ${ }^{1}$ Department of Pediatrics, Allergy, Immunology and Rheumatology, Guangzhou Women and Children's Medical Center, \\ Guangzhou Medical University and First Clinical Medical College, Jinan University, Guangzhou 510120; \\ ${ }^{2}$ Department of Pediatrics, The Third Affiliated Hospital of Sun Yat-Sen University, Guangzhou 510630; \\ ${ }^{3}$ Department of Rheumatology, Peking Union Medical College Hospital, \\ Chinese Academy of Medical Sciences, Beijing 100730, P.R. China
}

Received September 9, 2014; Accepted October 29, 2014

DOI: $10.3892 /$ br. 2014.380

\begin{abstract}
Reversible posterior leukoencephalopathy syndrome (RPLS) is a clinical syndrome based on changes in clinical imaging, and it has been reported to mainly occur in adults. However, it has been recently discovered that RPLS is also prevalent in infant patients, particularly in those using glucocorticoids, immunosuppressant medications and cytotoxic drugs. The current study presents a 5-year-old male with a previous diagnosis of systemic-onset juvenile idiopathic arthritis (SoJIA) and macrophage-activation syndrome who developed posterior reversible encephalopathy syndrome during treatment with glucocorticoids, disease-modifying antirheumatic drugs and biological agent (etanercept) therapy. After $\sim 5$ days of treatment, the patient made a complete clinical recovery; the magnetic resonance imaging reviewed 2 weeks later showed that the previous hyper-intensity signal had disappeared and the multiple lesions in the brain had been completely absorbed. The case report shows that, conforming to recent literature, SoJIA in infants should be considered a risk factor for developing RPLS. The clinical manifestations of the disease are multiple, but usually reversible, and the patients mostly have a good prognosis. Rapid diagnosis and treatment is essential as
\end{abstract}

Correspondence to: Professor Huasong Zeng, Department of Pediatric, Allergy, Immunology and Rheumatology, Guangzhou Women and Children's Medicial Center, Guangzhou Medical University and First Clinical Medical College, Jinan University, 402 Renmin Road, Guangzhou 510120, P.R. China

E-mail: huasongz@126.com

Abbreviations: SoJIA, systemic-onset juvenile idiopathic arthritis; RPLS, reversible posterior leukoencephalopathy syndrome; cyclosporine A, CsA; MTX, Methotrexate; MRI, magnetic resonance imaging; PRES, posterior reversible encephalopathy syndrome

Key words: systemic-onset juvenile idiopathic arthritis, reversible posterior leukoencephalopathy syndrome early treatment may prevent progression to irreversible brain damage. By increasing the awareness of RPLS, the patient care may improve and further insight may be gained.

\section{Introduction}

Reversible posterior leukoencephalopathy syndrome (RPLS) is a recently reported disorder present in a variety of clinical settings with characteristic findings in magnetic resonance imaging (MRI) exhibiting bilateral gray and white matter abnormalities in the posterior regions of the cerebral hemispheres and cerebellum (1). This syndrome is characterized by acute to subacute onset of headache, nausea, dizziness, changes in consciousness (lethargy, coma, diminished spontaneity of speech), convulsions and transient visual disturbances [such as cortical blindness (2)]. RPLS is caused by various heterogeneous factors and has been described in numerous medical conditions (3). Hypertension and immunosuppressive or cytotoxic drugs have been reported to be the most significant causes of RPLS in pediatric and adult patients $(4,5)$.

RPLS has been previously reported to mainly occur in adults, and is less frequent in children. However, it has been recently recognized that RPLS is also prevalent in child patients (6), particularly in those with acute leukemia, autoimmune disease (such as Henoch-Schönlein purpura or systemic lupus erythematosus), sickle cell anemia and nephrotic syndrome, in which patients were mainly taking glucocorticoids, immunosuppressant medications, cytotoxic drugs or had severe hypertension. The current study presents a case report of RPLS in a 5-year-old Chinese male with systemic-onset juvenile idiopathic arthritis (SoJIA) who was hospitalized in Guangzhou Women and Children's Medical Center (Guangzhou, China).

\section{Case report}

The study reports the case of a 5-year-old male who presented primarily with repeated joint pain (hip and knee), remittent fever ( 2 weeks), unfixed rash and pericardial effusion. 


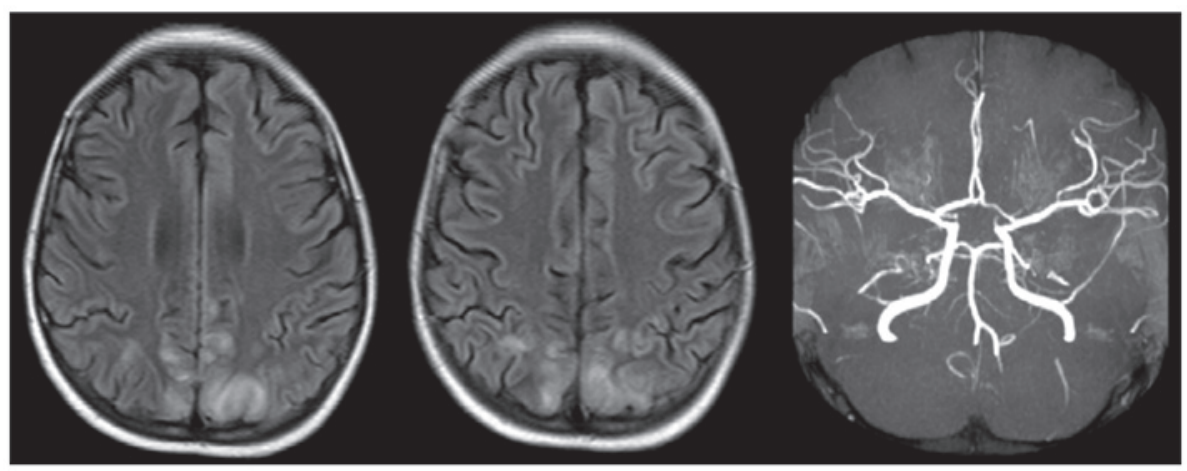

Figure 1. MRI axial T2WI image revealing cortical and subcortical hyper-intensities at the parieto-occipital regions (left two pictures) and MRA with display of the circle of Willis revealing the slender posterior cerebral artery (right picture). MRI, magnetic resonance imaging; T2WI, T2-weighted image; MRA, magnetic resonance angiography.

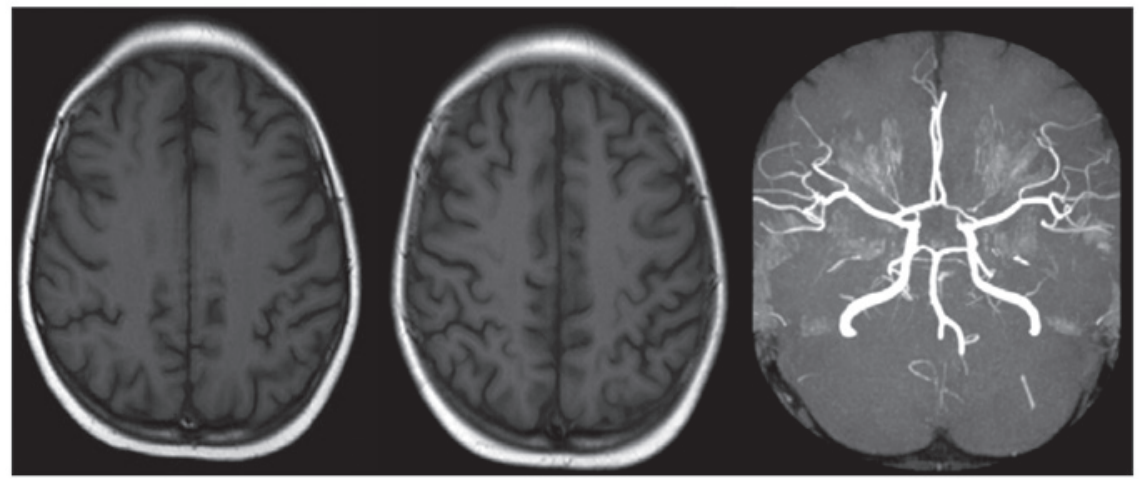

Figure 2. MRI scans obtained 2 weeks after onset. The previously existed hyper-intensity signal in the axial T2WI image had disappeared (left two pictures), MRA with display of the circle of wills revealing the slender posterior cerebral artery as before (right picture). MRI, magnetic resonance imaging; T2WI, T2-weighted image; MRA, magnetic resonance angiography.

Written informed consent was obtained from the patient for publication and any accompanying images. The patient was diagnosed to have SoJIA following exclusion of infectious diseases, leukemia and tumor diseases. Subsequently, the patient received treatment of glucocorticoids, cyclosporine A (CsA), Methotrexate (MTX) and a biological agent $(0.8 \mathrm{mg} / \mathrm{kg}$ etanercept per week injected hypodermicly), and the condition gradually improved. The patient was infected by the measles virus during the treatment process, and recovered following treatment with intravenous immunoglobulin.

However, after 2 months of treatment the patient suddenly presented with moderate hypertension, dizziness, apathia, diminished spontaneity of speech, exhibited consciousness changes and could only recognize his parents. Examination revealed severely impaired memory, dyscalculia and the ability of orientation decreased significantly. As opposed to certain other cases $(7,8)$, the patient had no convulsion and visual loss, and the neurological physical examination was normal. There was no clinical or laboratory evidence of infection, and the serum electrolyte levels were normal. At that time, blood pressure was $137 / 89 \mathrm{mmHg}$, the serum CsA level was $151.6 \mathrm{mg} / \mathrm{l}$ (therapeutic range, 120-200 mg/l). Subsequently, the patient received an emergency antihypertensive drug and the treatment for dehydration to lower the intracraninal pressure. A lumbar puncture and MRI were performed in order to exclude intracranial infections and thromboembolic disease. No clear abnormalities were detected in the cerebrospinal fluid. Magnetic resonance angiography (MRA) and diffusion-weighted imaging of the head (Fig. 1) showed a diffusely abnormal signal in the parieto-occipital areas, left hippocampus and left dorsal thalamus, T1-weighted image (T1WI) showed a low signal and T2WI showed a high signal, all of which was suggestive of typical RPLS imaging changes. In addition, MRA also found that the bilateral posterior cerebral artery was slender (Fig. 1). By antihypertensive treatment, and discontinued CsA treatment, the condition improved gradually during the first 3 days of hospitalization, as the patient could recall his friends' names and his speech was more fluent. All the symptoms disappeared completely on day 5 of hospitalization, and memory power, calculation ability and orientation ability of the patient had returned to premorbid levels, and the physical examination was normal with no discomfort. The MRI was reviewed 2 weeks later (Fig. 2) and showed that the previous hyper-intensity signal in T2WI had disappeared, the multiple lesions in the brain had been completely absorbed, whereas the posterior cerebral artery was as slender as before.

There have been over two months since the last MRI, and the patient regularly underwent a review in the outpatient department; blood pressure was controlled at $\sim 110 / 75 \mathrm{mmHg}$, and the current oral drugs included glucocorticoid, MTX and antihypertensive drugs, coupled with regular injections of 
biological agents. The patient currently has no discomfort and the condition remained stable.

\section{Discussion}

RPLS was first reported in 1996 by Hinchey et al (3), and represented a reversible, predominantly posterior leukoencephalopathy in patients that had renal insufficiency, hypertension or were maintained on immunosuppressants. RPLS is diagnosed based on the typical pattern of brain imaging and the reversibility of symptoms. With the recent wide-spread use of MRI in clinical practice, the relevant cases reported have increased every year; however, findings remain limited in pediatric patients. Radiographic lesions in posterior reversible encephalopathy syndrome (PRES) are rarely isolated to the 'posterior' parieto-occipital white matter and often involve the cortex, frontal lobes, basal ganglia and brainstem $(9,10)$. Although reversible by definition, if the patients did not undergo early recognition and timely treatment, irreversible brain damage may occur (11), and therefore, the restricted term of posterior reversible leukoencephalopathy may not be appropriate as the condition is not always reversible, it is not necessarily confined to the posterior regions of the brain and it can affect white and grey matter (12). Regarding this, Casey et al (2) suggested referring to RPLS as PRES. RPLS, or PRES, is characterized by acute to subacute disorder of headache, nausea, dizziness, changes in consciousness, convulsions and transient visual disturbances. The clinical manifestation varies, but not all symptoms appear in the whole process, and certain symptoms may occur repeatedly. Due to the previously reported low incidence and clinical features of reversibility, RPLS did not obtain enough clinical attention, and the pathogenesis remains unknown. Currently, the majority of studies (13-16) agree that RPLS usually occurs during the process of suddenly raised blood pressure or the use of certain drugs (including CsA, corticosteroids, other immunosuppressive and cytotoxic drugs). Abrupt hypertension contributes to the development of PRES, and the hyperperfusion theory is supported by the frequent presence of substantial hypertension in patients with PRES and subsequent resolution of clinical symptoms and radiological edema with prompt treatment of hypertension (2).

However, clinicians should also be aware that certain cases transpire in the absence of severe hypertension, and infants also develop RPLS at lower absolute pressures compared to adults. Unless pressures are recorded frequently and compared to baseline values, gradual increases or transient spikes in blood pressure may be overlooked. This applies to young or pregnant patients in particular, with baseline pressures that are normally low (9).

The pathophysiology of RPLS is complex, and there are various opinions on its pathogenesis $(3,19-21)$. Hypertension, cytotoxic drugs or other causes of RPLS are considered to induce endothelial damage and breakdown in cerebral autoregulation, which lead to the movement of fluid from the intravascular into the pericapillary astrocytes, as well as into the interstitium through the capillary wall $(3,21,22)$. Immunosuppressive and cytotoxic drugs, by direct endothelial damage or through the inhibition of prostacyclin, disrupt the endothelial cell integrity, causing them to release endothelin $(23,24)$, which causes vasoconstriction and vasospasm in cerebral vessels, initiates mild, reversible ischemia and white matter edema $(25,26)$. Specific binding sites for endothelin have been identified in several areas of the human brain, including the cerebellum, hippocampal formation, diencephalon and choroid plexus (25). Additionally, the cerebral white matter is composed of myelinated fibre tracts in a cellular matrix of glial cells, arterioles and capillaries, which make the structure more susceptible to the accumulation of fluid in the extracellular spaces (vasogenic oedema) (27), and is possibly the reason for the prone involvement of brain white matter. Regional variations in sympathetic innervation of the vasculature of the central nervous system may account for the unique localization of these lesions in the posterior cerebral hemispheres $(2,11)$. The study by Schwartz et al $(28)$ indicated that the vertebrobasilar system possesses sparse sympathetic innervation that may result in disruption of autoregulatory mechanisms, preferentially affecting the posterior lobes. Furthermore, glucocorticoids can also lead to increased blood pressure and endothelial dysfunction. In addition, previous studies (29-31) have reported that biological agents and measles virus infection or measles vaccination also cause RPLS, although the specific mechanism is unclear.

Although the prognosis is usually good, not quickly recognizing the syndrome and discontinuing the treatment with the offending agent may result in profound and permanent central nervous system dysfunction or fatality. Therefore, more attention should be focused on RPLS, particularly in patients with associated diseases or risk factors, and if the patients have a sudden episode of neurological symptoms, MRI examination should be performed in sufficient time. Early recognition of PRES is important for prompt therapy, which typically consists of gradual blood pressure control and withdrawal of the potentially offending agents (2), and of note, the MRI review following treatment is equally important for the diagnosis and treatment of this disease.

In a previous study, RPLS secondary to SoJIA has been reported in an infant with SoJIA during treatment with pulse methylprednisolone therapy (31), however, in this case the infant was younger and did not receive pulse glucocorticoids therapy, previous measles virus infection and the application of CsA may be the possible factors leading to RPLS, although the blood CsA concentration was within normal limits when RPLS occurred in this patient. Similar to the study (31), a sudden alteration in inflammatory milieu and/or hypertension in differing degrees of severity appear to be common to all the different implicated causes of PRES. The blood of the patient also had moderate hypertension at the onset of RPLS, whereas normal blood pressure was exhibited at ordinary times. In addition, with such a diffuse lesion scope in the head MRI, the study postulated that an increased vascular permeability associated with vascular endothelial damage may be the pathogenesis of PRES in this case, whereas the narrow brain artery ring in the patient may be only a congenital dysplasia.

The present case report highlights that there is also a possibly important association of RPLS with SoJIA. The early clinical manifestation of RPLS is various, and there may be no convulsions and no visual disturbances, but only apathia, moderate hypertension, diminished spontaneity of speech and retards in response. Early recognition of RPLS as a complication during various diseases and therapies in 
childhood may facilitate precise diagnosis and appropriate treatment, suggesting a good prognosis. In the present case, previous measles virus infection and the applications of CsA were possible factors leading to RPLS. Prompt diagnosis and therapy with antihypertensives, removal of the offending medication and treatment of the associated disorders is essential, as early treatment may prevent progression to irreversible brain damage. With increased medical awareness of RPLS, the patient care may be improved and further insight into this complication may be gained through continued research.

\section{Acknowledgements}

The authors would like to thank Dr Ying Xie and Dr Feng Li who contributed towards the article by making substantial contributions to conception, design, acquisition of data, or analysis and interpretation of data. Guangzhou City Grant (China) provided funding to Professor Huasong Zeng (no. 2012J4100031) for design, collection and decision to submit the manuscript for publication. The National High Technology Research and Development Program 863 (China) provided funding to Professor Xiaofeng Zeng (no. 012AA02A513) for the data analysis and writing of the manuscript.

\section{References}

1. Endo A, Fuchigami T, Hasegawa M, et al: Posterior reversible encephalopathy syndrome in childhood: report of four cases and review of the literature. Pediatr Emerg Care 28: 153-157, 2012.

2. Casey SO, Sampaio RC, Michel E, et al: Posterior reversible encephalopathy syndrome: utility of fluid-attenuated inversion recovery MR imaging in the detection of cortical and subcortieal lesions. AJNR Am J Neuroradiol 21: 1199-1260, 2000.

3. Hinchey J, Chaves C, Appignani B, et al: A reversible posterior leukoencephalopathy syndrome. N Engl J Med 334: 494-500, 1996.

4. Ikeda M, Ito S, Hataya H, Honda M and Anbo K: Reversible posterior leukoencephalopathy in a patient with minimal-change nephrotic syndrome. Am J Kidney Dis 37: E30, 2001.

5. Utsumi K, Amemiya S, Lizuka M, Lino Y and Katayama Y: Acute posterior leukoencephalopathy in a patient with nephrotic syndrome. Clin Exp Nephrol 7: 63-66, 2003.

6. Gümüs H, Per H, Kumandaş S and Yikilmaz A: Reversible posterior leukoencephalopathy syndrome in childhood: report of nine cases and review of the literature. Neurol Sci 31: 125-131, 2010.

7. Zhang YX, Liu JR, Ding MP, et al: Reversible posterior encephalopathy syndrome in systemic lupus erythematosus and lupus nephritis. Intern Med 47: 867-875, 2008.

8. Das CJ and Seith A: Posterior reversible encephalopathy syndrome (PRES). Indian Pediatr 43: 657-658, 2006.

9. Lee VH, Wijdicks EF, Manno EM, et al: Clinical spectrum of reversible posterior leukoencephalopathy syndrome. Arch Neurol 65: 205-210, 2008.

10. Bartynski WS and Boardman JF: Distinct imaging patterns and lesion distribution in posterior reversible encephalopathy syndrome. AJNR Am J Neuroradiol 28: 1320-1327, 2007.
11. Rajasekhar A and George TJ Jr: Gemcitabine-induced reversible posterior leukoencephalopathy syndrome: a case report and review of the literature. Oncologist 12: 1332-1335, 2007.

12. Stott VL, Hurrell MA and Anderson TJ: Reversible posterior leukoencephalopathy syndrome: a misnomer reviewed. Intern Med J 35: 83-90, 2005.

13. Yamamoto A, Hayakawa K and Houjyou M: CT and MRI findings of cyclosporine-related encephalopathy and hypertensive encephalopathy. Pediatr Radiol 32: 340-343, 2002.

14. Reinohs M, Straube T, Baum P, Berrouschot J and Wagner A: Recurrent reversible cerebral edema after long term immunosuppression with tacrolimus. J Neurol 249: 780-781, 2002.

15. Bhatt A,FarooqMU,Majid A and KassabM:Chemotherapy-related posterior reversible leukoencephalopathy syndrome. Nat Clin Pract Neurol 5: 163-169, 2009.

16. Ishikura K, Ikeda M, Hamasaki Y, et al: Nephrotic state as a risk factor for developing posterior reversible encephalopathy syndrome in paediatric patients with nephrotic syndrome. Nephrol Dial Transplant 23: 2531-2536, 2008

17. Fugate JE, Claassen DO, Cloft HJ, Kallmes DF, Kozak OS and Rabinstein AA: Posterior reversible encephalopathy syndrome: associated clinical and radiologic findings. Mayo Clin Proc 85: 427-432, 2010.

18. Pavlakis SG, Frank Y, Kalina P, et al: Occipital-parietal encephalopathy: a new name for an old syndrome. Pediatr Neurol 16: 145-148, 1997.

19. Garg RK: Posterior leukoencephalopathy syndrome. Postgrad Med J 77: 24-28, 2001.

20. Schwartz RB: Hyperperfusion encephalopathies: hypertensive encephalopathy and related conditions. Neurologist 8: 22-34, 2002.

21. Kwon S, Koo J and Lee S: Clinical spectrum of reversible posterior leukoencephalopathy syndrome. Pediatr Neurol 24: 361-364, 2001.

22. Singh N,Bonham A and Fukui M: Immunosuppressive-associated leukoencephalopathy in organ transplant recipients. Transplantation 69: 467-472, 2000.

23. Gijtenbeek JM, van den Bent MJ and Vecht CJ: Cyclosporine neurotoxicity: a review. J Neurol 246: 339-346, 1999.

24. Truwit CL, Denaro CP, Lake JR and DeMareo T: MR imaging of reversible cyclosporine A-induced neurotoxicity. AJNR Am J Neuroradiol 12: 651-659, 1991.

25. Vlasveld LT, Cornelissen JJ, Dellemijn PL, Joosse MV and van den Bent MJ: Cortical blindness during treatment with cyclosporine. Ned Tijdschr Geneeskd 138: 2057-2061, 1994 (In Dutch).

26. Garg RK: Posterior leukoencephalopathy syndrome. Postgrad Med J 77: 24-28, 2001.

27. Schwartz RB, Bravo SM, Klufas RA, et al: Cyclosporine neurotoxicity and its relationship to hypertensive encephalopathy: CT and MR findings in 16 cases. AJR AM J Roentgennol 165: 627-631, 1995.

28. Hamano T, Takeda T, Morita $\mathrm{H}$, et al: Posterior reversible encephalopathy syndrome following measles vaccination. J Neurol Sci 298: 124-126, 2010.

29. Rebora AE and Regesta G: Reversible posterior leukoencephalopathy: a possible threat for psoriatic patients treated with biological agents. J Eur Acad Dermatol Venereol 24: 97-98, 2010.

30. Mavragni CP, Vhchoyiannopoulos PC, Kosmas N, et al: A case of reversible posterior leucoencephalopathy syndrome after rituximab infusion. Rheumatology (Oxford) 43: 1450-1451, 2004.

31. Kumar S and Rajam L: Posterior reversible encephalopathy syndrome (PRES/RPLS) during pulse steroid therapy in macrophage activation syndrome. Indian J Pediatr 78: 1002-1004, 2011. 